University of Nebraska - Lincoln

DigitalCommons@University of Nebraska - Lincoln

Faculty Publications, Department of Physics and Astronomy

Research Papers in Physics and Astronomy

4-15-1999

Dielectric tensor for magneto-optic NiMnSb

\author{
Xiang Gao \\ University of Nebraska-Lincoln \\ John A. Woollam \\ University of Nebraska-Lincoln, jwoollam1@unl.edu \\ Roger D. Kirby \\ University of Nebraska-Lincoln, rkirby1@unl.edu \\ David J. Sellmyer \\ University of Nebraska-Lincoln, dsellmyer@unl.edu \\ C. T. Tanaka \\ Massachusetts Institute of Technology \\ See next page for additional authors
}

Follow this and additional works at: https://digitalcommons.unl.edu/physicsfacpub

Part of the Physics Commons

Gao, Xiang; Woollam, John A.; Kirby, Roger D.; Sellmyer, David J.; Tanaka, C. T.; Nowak, J.; and Moodera, Jagadeesh S., "Dielectric tensor for magneto-optic NiMnSb" (1999). Faculty Publications, Department of Physics and Astronomy. 42.

https://digitalcommons.unl.edu/physicsfacpub/42

This Article is brought to you for free and open access by the Research Papers in Physics and Astronomy at DigitalCommons@University of Nebraska - Lincoln. It has been accepted for inclusion in Faculty Publications, Department of Physics and Astronomy by an authorized administrator of DigitalCommons@University of Nebraska Lincoln. 


\section{Authors}

Xiang Gao, John A. Woollam, Roger D. Kirby, David J. Sellmyer, C. T. Tanaka, J. Nowak, and Jagadeesh S. Moodera 


\title{
Dielectric tensor for magneto-optic NiMnSb
}

\author{
Xiang Gao and John A. Woollam \\ Center for Microelectronic and Optical Materials Research, and Department of Electrical Engineering, University of Nebraska, \\ Lincoln, Nebraska 68588-0511 \\ R. D. Kirby and D. J. Sellmyer \\ Behlen Laboratory of Physics and Center for Materials Research and Analysis, University of Nebraska, Lincoln, Nebraska 68588 \\ C. T. Tanaka, J. Nowak, and J. S. Moodera \\ Francis Bitter Magnet Laboratory, Massachusetts Institute of Technology, Cambridge, Massachusetts 02139
}

(Received 24 August 1998; revised manuscript received 9 December 1998)

\begin{abstract}
The diagonal component $\varepsilon_{x x}$ and off-diagonal component $\varepsilon_{x y}$ of the complex dielectric tensor for the ferromagnetic compound $\mathrm{NiMnSb}$ are determined using ex situ spectroscopic ellipsometry and magneto-optic analysis over the spectral range from 0.7 to $6.2 \mathrm{eV}$. The effects of the overcoat on the raw data are removed by the analysis. First, the complex $\varepsilon_{x x}$ of thin-film NiMnSb were determined by ex situ spectroscopic ellipsometry; then $\varepsilon_{x y}$ was determined by analyzing Kerr rotation and ellipticity data using the determined $\varepsilon_{x y}$ data. Lorentz oscillators were used to model peaks seen in the $\varepsilon_{x x}$ spectra. The diagonal dielectric component $\varepsilon_{x x}$ is dominated by free-carrier effects below $1.15 \mathrm{eV}$, and dominated by interband transitions above $2.0 \mathrm{eV}$. The center energies of the Lorentz oscillators are consistent with the calculated band structure and minority-spin optical conductivity of NiMnSb. Joint density of states and optical conductivity calculated from $\varepsilon_{x x}(2)$ data with free-carrier effects removed shows onset energies at $\sim 0.6$ and $\sim 0.2 \mathrm{eV}$, respectively. From a study of the $\varepsilon_{x x}$ and $\varepsilon_{x y}$ spectra, the Kerr rotation peak at lower energy is determined to be due to combined contributions from: (1) a crossover between the free-carrier effect and interband transitions, and (2) transitions involving spin-orbit coupling. The high-Kerr rotation peaks at higher energies result exclusively from transitions involving spin-orbit coupling. [S0163-1829(99)13615-4]
\end{abstract}

\section{INTRODUCTION}

Ferromagnetic compounds like $\mathrm{NiMnSb}$ and $\mathrm{PtMnSb}$ have been studied as magneto-optic (MO) materials for more than a decade. ${ }^{1-20}$ Both compounds are predicted to be halfmetallic materials. ${ }^{2}$ The half-metallic nature was confirmed by spin-polarized measurements (positron annihilation studies) for $\mathrm{NiMnSb},{ }^{9}$ and transport measurements for $\mathrm{NiMnSb}$ and PtMnSb. ${ }^{8,11}$ Accurate values for diagonal and offdiagonal components of the dielectric tensor are important for magneto-optic studies as well as band-structure studies. The $\varepsilon_{x x}$ spectrum (diagonal part of the dielectric tensor) for $\mathrm{NiMnSb}$ was published for bulk materials, ${ }^{4}$ and the offdiagonal part $\varepsilon_{x y}$ was derived from the Kerr responses (rotations and ellipticities) and the $\varepsilon_{x x}$ spectrum. ${ }^{6}$ Smaller Kerr rotations and ellipticities were found for NiMnSb compared to PtMnSb. ${ }^{1}$ Different possible origins of the Kerr responses have been suggested in spin-orbit coupling, ${ }^{3}$ plasma-edge enhancement, ${ }^{5,16}$ and scalar relativistic effects. ${ }^{7} \mathrm{~A} b$ initio theoretical calculations have been performed for $\varepsilon_{x x}$, and $\varepsilon_{x y} \cdot{ }^{12-16}$ Kerr rotation and ellipticity were in turn calculated and compared to available experimental data.

Recently, thin-film NiMnSb has attracted considerable interest due to its half-metallic nature-metallic for majorityspin electrons and semiconductive for minority-spin electrons-which leads to the $100 \%$ spin polarization at the Fermi level. This means it potentially can be used as "spin filter" layers in multilayer systems to produce stronger conventional or tunneling giant magneto-resistance effects. ${ }^{10,17-20}$ Large magnetoresistance was found in evaporated $\mathrm{NiMnSb} / \mathrm{Al}_{2} \mathrm{O}_{3} / \mathrm{NiFe}$ multilayer structures due to a spin-polarized tunneling effect. ${ }^{19}$ MO Kerr responses over the range from 0.8 to $5.0 \mathrm{eV}$ for sputter-deposited $\mathrm{NiMnSb}$ thin films have been published. ${ }^{20}$ Also, the diagonal part of the dielectric tensor (in terms of complex optical constants $n$ and $k$ ) for sputter deposited thin-film NiMnSb and PtMnSb were measured in the spectral range from 1.4 to $5 \mathrm{eV} .{ }^{18}$

In this paper, a precise method for measuring the dielectric tensor of thin-film NiMnSb over the range from 0.7 to $6.2 \mathrm{eV}$ is used: a combination of ex situ spectroscopic ellipsometry (SE) and magneto-optic analysis, based on electromagnetic theory, ${ }^{21,22}$ which works effectively for multilayer structures. ${ }^{23}$ Since NiMnSb easily oxidizes, direct measurements of both the diagonal and the off-diagonal parts of its dielectric tensor are not possible in air. Multilayer analysis on samples with a protective cover layer is necessary to precisely determine the intrinsic dielectric tensor of the underlying magnetic materials.

Results are compared with recent calculations of $\varepsilon_{x x}$, $\sigma_{x y}(2)$, and calculated band structure by V. N. Antonov et al. ${ }^{15}$ Further, the spectrally dependent $\varepsilon_{x x}$ data are fitted in regression analysis using a phenomenological Lorentz oscillator model with four oscillators plus a Drude term. The fits are excellent, and the relative contributions of interband transitions and free-carrier effects (Drude) to $\varepsilon_{x x}$ are determined. Optical conductivity and joint density of states (JDOS) were then calculated exclusively for the four oscillators, and the results are compared to results of Ref. 4. 


\section{THEORY}

A Jones matrix $J$ is used to describe the optical system for MO material structures, given by ${ }^{21,22}$

$$
\mathbf{J}=\left(\begin{array}{ll}
R_{x} & K_{y} \\
K_{x} & R_{y}
\end{array}\right) .
$$

The reflected electric field $E_{r}$ is related to the incident electric field $E_{i}$ by the Jones matrix: $\mathbf{E}_{r}=\mathbf{J} \cdot \mathbf{E}_{i}$. Kerr rotation and ellipticity are defined by: $\theta_{k}=\operatorname{Re}\left(K_{x} / R_{x}\right)$ and $\eta_{k}$ $=\operatorname{Im}\left(K_{x} / R_{x}\right)$, respectively. In this paper where the incident light beam is at normal angle, the Jones matrix is a function of wavelength, the individual layer thicknesses, and the spectrally dependent dielectric tensors of the individual layers. With layer thicknesses and measuring wavelengths predetermined, the dielectric tensor for each individual layer is the only parameter to be determined.

For an MO layer, the dielectric tensor is given by

$$
\varepsilon=\left(\begin{array}{ccc}
\varepsilon_{x x} & \varepsilon_{x y} & 0 \\
-\varepsilon_{x y} & \varepsilon_{x x} & 0 \\
0 & 0 & \varepsilon_{x x}
\end{array}\right) .
$$

The magneto-optic Kerr response is determined by both diagonal and off-diagonal components in the dielectric tensor. By normalizing Eq. (2) the dielectric tensor can be expressed as

$$
\varepsilon=\varepsilon_{x x}\left(\begin{array}{ccc}
1 & -i Q & 0 \\
i Q & 1 & 0 \\
0 & 0 & 1
\end{array}\right) \text {, }
$$

where $Q$ (Voigt parameter) is defined as

$$
Q=Q_{1}+i Q_{2}=i \varepsilon_{x y} / \varepsilon_{x x} .
$$

Here, "1" and " 2 "' denote the real and imaginary parts of the complex $Q$. This will be the same for complex $\varepsilon_{x x}$ and $\varepsilon_{x y}$.

To determine the dielectric tensor, ex situ spectroscopic ellipsometry analysis was first used to measure the ellipsometric parameters $\Psi$ and $\Delta$, and $\varepsilon_{x x}$ was then found by regression fits to the $\mathrm{SE}$ data. In our analysis, the dielectric function $\varepsilon_{x x}$ for materials other than $\mathrm{NiMnSb}\left[\mathrm{MgO},{ }^{24}\right.$ vanadium, ${ }^{25}$ and $\mathrm{Al}_{2} \mathrm{O}_{3}$ (Ref. 26)] in the multilayer structure were taken as fixed, using published data, and the thicknesses for all layers were predetermined during molecularbeam epitaxy growth. Thus, the $\varepsilon_{x x}$ for $\mathrm{NiMnSb}$ is the only parameter necessary to be fitted for in the regression, and may be precisely determined without correlation to other variables.

Secondly, since $\varepsilon_{x x}$ has been determined and $Q$ values are zero for the nonmagnetic layers in the analyzed multilayer structures, $Q$ for the NiMnSb magnetic layer can be measured using a simple procedure similar to that for finding $\varepsilon_{x x}$ : regression fitting $Q$ to the measured magneto-optic Kerr rotation and ellipticity data. Here, $Q$ for NiMnSb is the only variable; therefore, it is also determined precisely and with no correlation to other variables. $\varepsilon_{x y}$ is then determined using Eq. (4) from the determined $\varepsilon_{x x}$ and $Q$ values.
A phenomenological Lorentz oscillator (LO) model was used to fit the diagonal dielectric tensor element $\varepsilon_{x x}$. The LO model is given by ${ }^{27}$

$$
\varepsilon_{x x}=\varepsilon_{\infty}+\sum_{j} \frac{A_{j}}{\left(E_{j}^{2}-E^{2}\right)-i E \cdot B_{j}},
$$

where $A_{j}=4 \pi n e^{2} \hbar / m_{j}^{*}, \quad B_{j}=\hbar / \tau_{j}, \quad E_{j}=\hbar \omega_{j}$, and $E$ $=\hbar \omega . \quad E_{j}$ is the center energy of oscillator $j$, which could potentially correspond to one of the critical-point transitions of the material. More likely, each oscillator might be due to an integrated combination of transitions from different parts of the band structure. In any case, such a phenomenological analysis should be helpful to theorists in making comparisons with experiment. In the oscillator analysis $\varepsilon_{\infty}$ is a constant. $\quad m_{j}^{*}$ is the effective mass and $\tau_{j}$ the relaxation time of oscillator $j . \quad n$ is the carrier density for the material and $e$ is the electron charge. $A_{j}$ and $B_{j}$ are defined as the amplitude and the broadening factor of each individual oscillator, and $A_{j}, B_{j}, E_{j}$, plus $\varepsilon_{\infty}$ are the fit parameters in the regression. When $E_{j}$ is zero, the corresponding oscillator has the same form as the classic Drude model for free carriers.

The dielectric tensor can be converted to the conductivity tensor through

$$
\begin{gathered}
\sigma_{x x}=i \omega\left(1-\varepsilon_{x x}\right) / 4 \pi, \\
\sigma_{x y}=-i \omega \varepsilon_{x y} / 4 \pi .
\end{gathered}
$$

We compare the $\omega \sigma_{x y}(2)$ spectra to the calculated spectra published in Ref. 15.

\section{EXPERIMENTAL RESULTS AND ANALYSIS}

\section{A. Optical spectra}

The single-crystal NiMnSb sample was grown on a (100) $\mathrm{MgO}$ substrate at $200^{\circ} \mathrm{C}$ by molecular-beam epitaxy. The $\mathrm{NiMnSb}$ layer was sandwiched between a vanadium seed layer and an $\mathrm{Al}_{2} \mathrm{O}_{3}$ protective layer. The atomic composition ratio for $\mathrm{Ni}: \mathrm{Mn} \mathrm{Sb}$ is 33.8:33.2:33.0, determined from Rutherford back-scattering spectroscopy. Layer thicknesses for $V$, $\mathrm{NiMnSb}$, and $\mathrm{Al}_{2} \mathrm{O}_{3}$ layers are 5, 83.4, and $4 \mathrm{~nm}$, respectively, which are determined using an in situ quartz-crystal monitor with $\pm 1 \%$ precision.

Ex situ SE data are shown in Fig. 1(a) taken with three different incidence angles. The dielectric function $\varepsilon_{x x}$ of the $\mathrm{NiMnSb}$ layer was then determined by regression fitting for $\varepsilon_{x x}$ from the SE data, as described in the previous section. During the fit, published values of the dielectric functions for $\mathrm{MgO}, \mathrm{V}$, and $\mathrm{Al}_{2} \mathrm{O}_{3}$ were used, ${ }^{24-26}$ with thicknesses of the layers fixed at predetermined values. The $\varepsilon_{x x}$ data for NiMnSb are shown in Fig. 2, as well as published theoretical $\varepsilon_{x x}$ data for NiMnSb (Ref. 15).

By inspection of the $\varepsilon_{x x}$ spectra, a Drude edge is seen in the lower energy range. However, in the higher energy range, the features are more semiconductorlike. Comparing experimental $\varepsilon_{x x}$ data in Fig. 2 to previously published theoretical results (Ref. 15), reasonably good agreement (especially in the real part of $\varepsilon_{x x}$ ) is found with respect to the peak positions, which are at roughly the same photon energies. However the peaks in the experimental data are generally much 

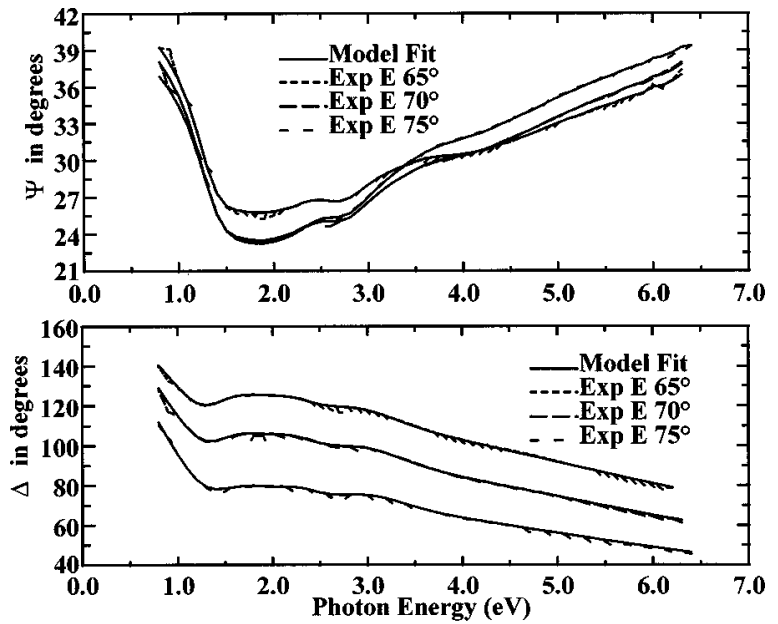

(a)

\begin{tabular}{|llr|}
\hline 3 & $\mathrm{Al2O}$ & $4 \mathrm{~nm}$ \\
\hline 2 & $\mathrm{NiMnSb}$ & $83.4 \mathrm{~nm}$ \\
\hline 1 & $\mathrm{~V}$ & $5 \mathrm{~nm}$ \\
\hline 0 & $\mathrm{MgO}$ & $1 \mathrm{~mm}$ \\
\hline
\end{tabular}

(b)

FIG. 1. (a) Experimental SE data taken from the sample with the structure shown in (b) and the regression model fit for $\varepsilon_{x x}$ of the $\mathrm{NiMnSb}$ layer. The published $\varepsilon_{x x}$ data were used for $\mathrm{MgO}$ (Ref. 24), vanadium (Ref. 25), and $\mathrm{Al}_{2} \mathrm{O}_{3}$ (Ref. 26).

weaker and smoother. Differences are probably due to: (1) the imperfections (defects and grain boundaries) in the experimentally grown layers; (2) the thermal conditions (room temperature measurements); (3) roughness at the surfaces and interfaces of the experimental materials; and (4) the finite experimental thickness for the NiMnSb compared to the infinite bulk crystal used in theoretical calculations.

To quantify the contributions from both the free-carrier effect and the interband transitions, the $\varepsilon_{x x}$ data were fit
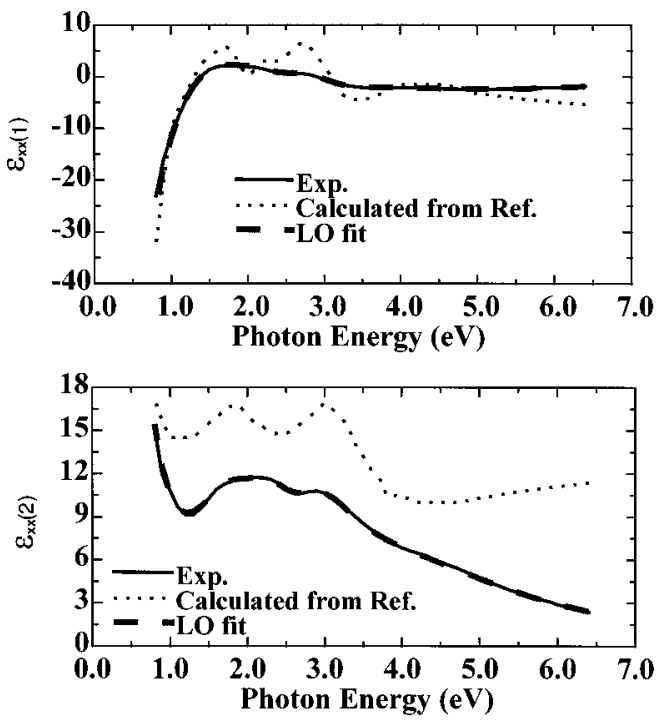

FIG. 2. Diagonal dielectric component $\varepsilon_{x x}$ of the NiMnSb layer obtained from the fit of Fig. 1 and the Lorentz oscillator fit for the $\varepsilon_{x x}$. Also shown are the theoretically calculated data duplicated from Ref. 15 .
TABLE I. The Lorentz oscillator fit results for $\varepsilon_{x x}$ of NiMnSb. (90\% confident limits are shown behind the " \pm " signs)

\begin{tabular}{lccc}
\hline \hline $\begin{array}{c}\varepsilon x(1) \text { (inf.) } \\
1.23 \pm 0.04 \\
\text { Center Energy } \\
(\mathrm{eV})\end{array}$ & $\begin{array}{c}\text { Amp. } \\
(\mathrm{eV})\end{array}$ & $\begin{array}{c}\text { Broadening factor } \\
(\mathrm{eV})\end{array}$ \\
\hline Oscillator \#0 & 0 (Drude) & $7.1 \pm 0.1$ & $0.27 \pm 0.01$ \\
$\# 1$ & $1.77 \pm 0.02$ & $9.43 \pm 1.36$ & $1.02 \pm 0.05$ \\
$\# 2$ & $2.28 \pm 0.01$ & $7.42 \pm 1.56$ & $0.88 \pm 0.09$ \\
$\# 3$ & $3.05 \pm 0.01$ & $14.3 \pm 2.5$ & $1.16 \pm 0.08$ \\
$\# 4$ & $4.42 \pm 0.07$ & $103 \pm 5$ & $4.62 \pm 0.15$ \\
\hline \hline
\end{tabular}

using the LO model [Eq. (5)]. The fit is also shown in Fig. 2 and results of the Lorentz parameters are given in Table I. The model fit was excellent with one Drude term and four oscillators. It can be seen in the figure that the experimental data and LO fits are overlapped. The Drude term has a larger amplitude and much smaller broadening factor compared to the four other oscillators in the model. Center energies for the other oscillators are $1.77,2.28,3.05$, and $4.42 \mathrm{eV}$, respectively. Using more/fewer oscillators was tried, but the "goodness of fit", parameter was always worse. Furthermore, the correlation coefficients among floating oscillator parameters of the fits for the five oscillators were all acceptably low (high correlation indicates lack of uniqueness). The degrees of uncertainty (from the $90 \%$ confidence limits) on each parameter, provided by the regression procedure, are listed in Table I. Note that the uncertainties on the center energies are low for all five oscillators. The uncertainties on the amplitudes of four oscillators are higher than other parameters, but generally uncertainties are low.

Comparison of contributions from the free-carrier effect and other oscillators are shown in Fig. 3. One can see from the figure that the Drude effect dominates in the lower energy range and the oscillator effects dominate in the higher energy range. The crossover energy between the Drude effect and the interband transitions is at $1.15 \mathrm{eV}$ in the $\varepsilon_{x x}(2)$ spectra. The Drude effect approaches zero with increasing photon energies in both the $\varepsilon_{x x}(1)$ and $\varepsilon_{x x}(2)$ spectra; meanwhile, the oscillator contributions to the $\varepsilon_{x x}(1)$ decrease to zero with increasing energies, but the contributions to $\varepsilon_{x x}(2)$ are significantly higher in the higher energy range.

The crossover between the Drude contribution and the oscillator contributions creates positive $\varepsilon_{x x}(1)$ data over the range from $\sim 1.4$ to $\sim 2.5 \mathrm{eV}$, and also creates a minimum in $\varepsilon_{x x}(2)$ at $\sim 1.3 \mathrm{eV}$. This result has a significant effect on the Kerr spectra, which will be discussed in Sec. III B.

The optical conductivity $\sigma$ and joint density of states for interband transitions were calculated from the $\varepsilon_{x x}(2)$ data determined by LO parameters (excluding the Drude term) over the spectral range from 0.02 to $6.2 \mathrm{eV}$, as shown in Fig. 4. The relation between JDOS and $\varepsilon_{x x}(2)$ is given by ${ }^{4}$

$$
\mathrm{JDOS}=\int d k \delta\left[E_{f}(k)-E_{i}(k)-\hbar \omega\right]=\omega^{2} \varepsilon_{x x}(2),
$$

where $f$ an $i$ denote the final and initial states, and the relation between $\sigma$ and $\varepsilon_{x x}(2)$ is given by 


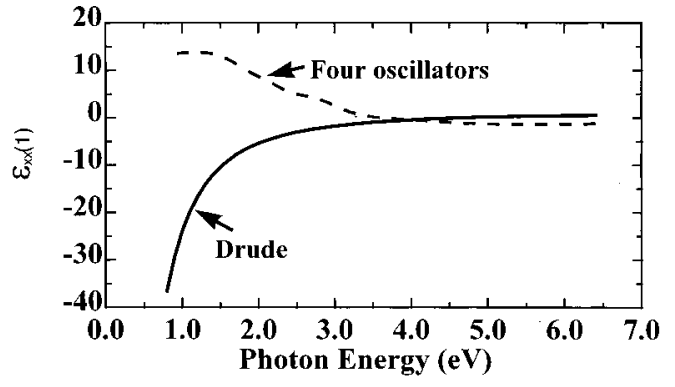

(a)

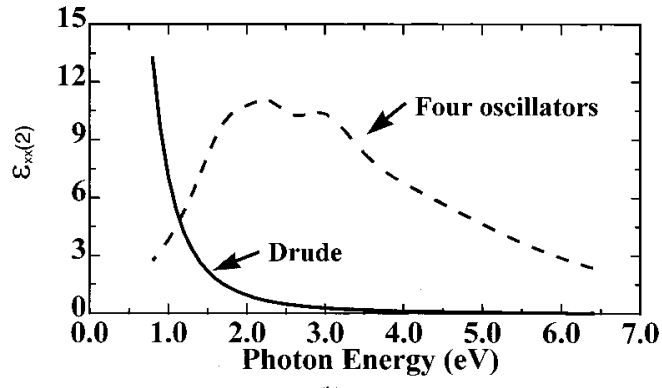

(b)
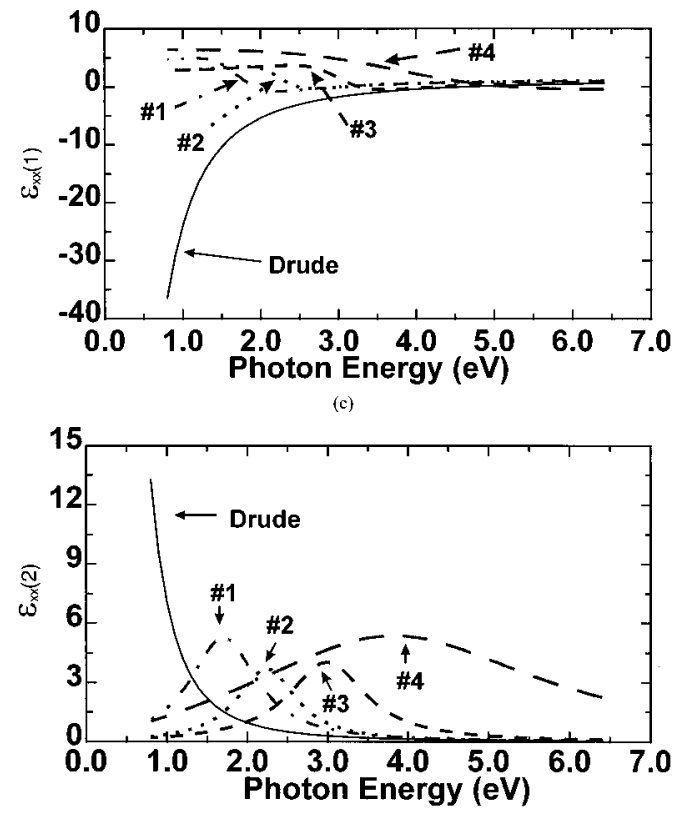

(d)

FIG. 3. The Drude term (solid line), total oscillator contribution (a) and (b), and separated oscillators contributions (c) and (d) for $\varepsilon_{x x}$ of the NiMnSb layer.

$$
\sigma=\omega \varepsilon_{x x}(2)
$$

The relatively broadened onset energy for $\sigma$ from our work is $\sim 0.2 \mathrm{eV}$. This is smaller than the $0.7 \mathrm{eV}$ onset energy for $\sigma$ determined in Ref. 4 . The difference is due mainly to the fact in Ref. 4 the Drude contribution was obtained by fitting experimental $\sigma$ data only in a small energy range instead of over the entire wide energy range used in this work. The result reported in Ref. 4 is a larger Drude-effect contribution and a smaller interband-transition contribution to the optical conductivity values, which in turn leads to a higher onset energy than found in the present paper.

The onset energy for the JDOS is $\sim 0.6 \mathrm{eV}$ from our results, as can be observed in Fig. 4.

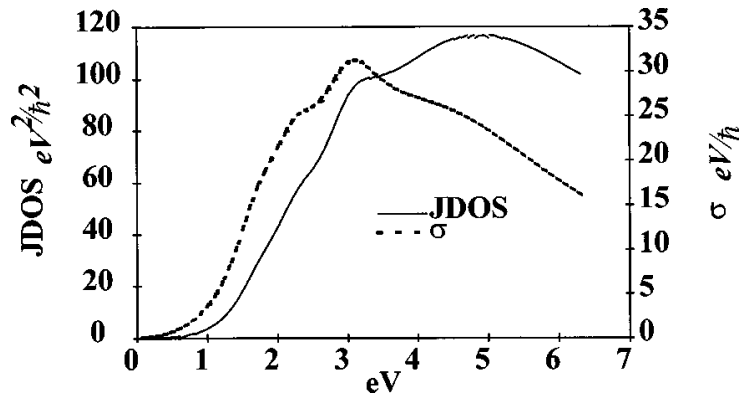

FIG. 4. The joint density of states and the optical conductivity $\sigma$ calculated from the four oscillators obtained from the LO fit, excluding the free-carrier Drude effect.

The corresponding direct interband transitions at 1.8, 2.2, and $3.1 \mathrm{eV}$ (for oscillators \#1, \#2, and \#3, respectively) involve the $L$ point and $\Gamma$ point from the calculated relativistic, spin-polarized energy band structure for $\mathrm{NiMnSb}$ published in Ref. 15. Sharp density of states peaks were seen in both sides of the Fermi level for 2.2 and $3.1 \mathrm{eV}$ transitions. For the $1.8 \mathrm{eV}$ transition, a peak in the upper band, and a shoulder in the lower band were seen. Oscillator \#4 is so broadened that it has less meaning in a band-gap correspondence.

The maximum in the minority-spin optical conductivity spectrum shown in Ref. 4 (calculated from the band structure given in Ref. 2) has peaks near $1.95 \mathrm{eV}$ (extends from 1.8$2.3 \mathrm{eV}$ ) and near $3.03 \mathrm{eV}$ (extends from $2.4-3.7 \mathrm{eV}$ ). This is also consistent with our results.

It should be pointed out that in Ref. 18, the author suggested a $1.1 \mathrm{eV}$ band gap for NiMnSb from the onset peak position of the transmission spectrum. Transmission data were generated from our results using the LO model for a 40-nm thick NiMnSb layer (the nominal thickness from Ref. 18 ), producing a very similar spectrum except for an apparent onset energy at $1.3 \mathrm{eV}$. That is to say, the transmission peak at lower energy is due to the crossover between the broadening of the interband transitions at higher energy and the onset of free-carrier effects at lower energy. This result demonstrates that it is inadequate to obtain the band gap from the onset energy of the transmission data. The freecarrier Drude-effect dominance in the lower energy range makes it impossible to see the band gap for NiMnSb, which is predicted to be indirect at $\sim 0.6 \mathrm{eV}$. ${ }^{15}$

Conclusions from LO model fits are as follows: The broadened interband transitions contribute to the $\varepsilon_{x x}$ spectrum in the range from $<0.7$ to $>6.2 \mathrm{eV}$. The oscillator positions are consistent with the band structure calculated in Ref. 15, and with the predicted minority-spin optical conductivity in Ref. 4. The free-carrier Drude effect dominates in the lower energy range and interband transitions dominate in the higher energy range.

\section{B. Magneto-optic spectra}

By modifying the same ellipsometer used for ex situ spectroscopic ellipsometry, MO polar Kerr rotation and ellipticity data were taken over the same spectral range. However, Kerr response signals became much noisier in the UV range. Therefore only the best data (from 0.7 to $5.4 \mathrm{eV}$ ) are presented. Kerr responses are shown in Fig. 5. The MO analysis was also done using a regression fit, as described in the 

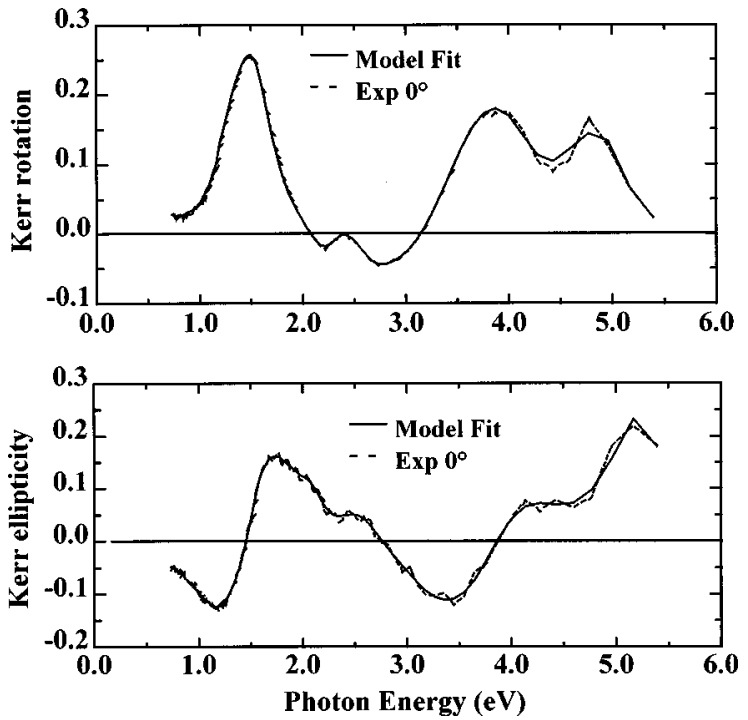

FIG. 5. Kerr rotation and ellipticity data taken for the $\mathrm{NiMnSb}$ sample, and the fit for the complex $Q$ values for the magnetic $\mathrm{NiMnSb}$ layer.

theory section. As a result, the Voigt parameter was determined, as shown in Fig. 6. The off-diagonal component $\varepsilon_{x y}$ for the same NiMnSb layer was then calculated using Eq. (4) and is shown in Fig. 7.

The off-diagonal conductivity component $\sigma_{x y}$ for $\mathrm{NiMnSb}$ was converted from $\varepsilon_{x y}$ using Eq. (6) and the imaginary part of the $\omega \sigma_{x y}$ spectrum $\omega \sigma_{x y}(2)$ was compared to the theoretically calculated $\omega \sigma_{x y}(2)$ spectrum published in Ref. 15 over the same spectral range, as shown in Fig. 8. There is rough qualitative agreement. However, the experimental data have more peaks in the spectra, and the maximum position in the low-energy range is $1.6 \mathrm{eV}$, compared to $2.1 \mathrm{eV}$ calculated in Ref. 15. In the higher energy range, two peaks $(\sim 3.9$ and $\sim 4.8 \mathrm{eV})$ were found compared to one at $\sim 4 \mathrm{eV}$ in the calculated spectrum. The negative points in the experimental $\omega \sigma_{x y}(2)$ data correspond to the zero crossing in the Kerr-rotation spectrum. (This is also seen in previously published Kerr responses for NiMnSb. ${ }^{1,20}$ ) However, the zero crossing is not seen in the theoretically calculated Kerr rotation (Ref. 15).

Comparing $\varepsilon_{x y}$ (Fig. 7) or $\omega \sigma_{x y}$ (2) (Fig. 8), to $\varepsilon_{x x}$ (Fig. 2) reveals that more, and sharper peaks are found in the $\varepsilon_{x y}$ spectra than in the $\varepsilon_{x x}$ spectra between 1 and $6 \mathrm{eV}$, espe-

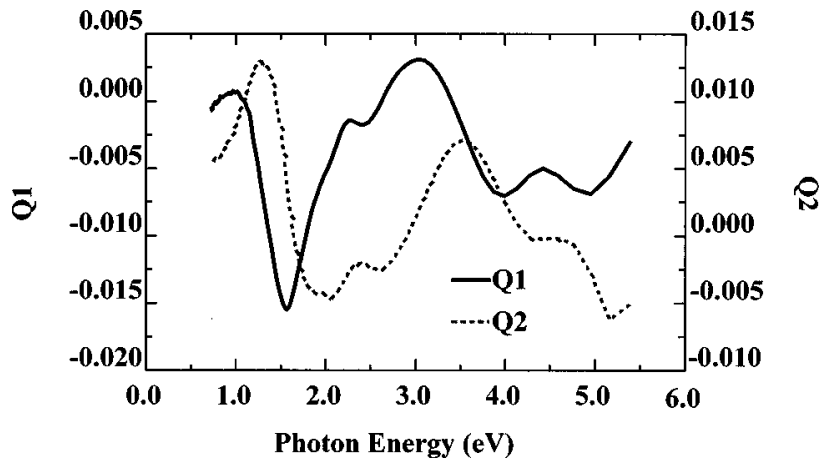

FIG. 6. The measured Voigt parameter $Q$ for $\mathrm{NiMnSb}$.

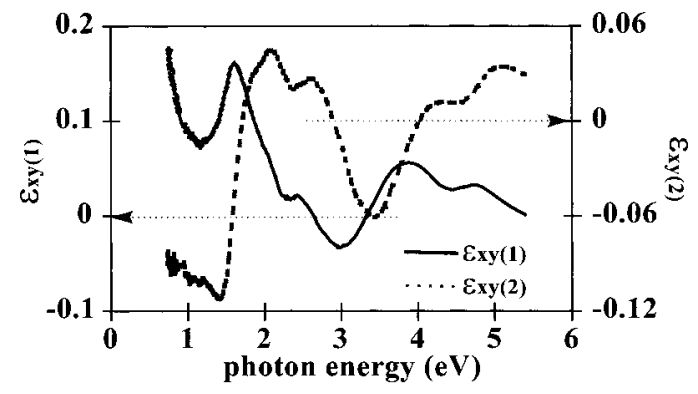

FIG. 7. Off-diagonal dielectric component $\varepsilon_{x y}$ for the $\mathrm{NiMnSb}$ layer.

cially at $\sim 3.9$ and $\sim 4.8 \mathrm{eV}$ where the $\varepsilon_{x x}$ spectra are flat. The peaks in the $\varepsilon_{x y}$ spectra come from transitions involving spin-orbit coupling. ${ }^{28,29}$

The relationship between Kerr rotation $\theta_{k}$, ellipticity $\eta_{k}$, $\varepsilon_{x y}$, and $\varepsilon_{x y}$ is given by

$$
\theta_{k}+i \eta_{k}=-\varepsilon_{x y} /\left[\left(\varepsilon_{x x}-1\right) \varepsilon_{x x}^{1 / 2}\right]
$$

The largest Kerr rotation peak is at $\sim 1.5 \mathrm{eV}$, as shown in Fig. 5. One can see that maxima can be found in both $\varepsilon_{x y}(1)$ and $\varepsilon_{x y}(2)$ spectra around $\sim 1.5 \mathrm{eV}$, as shown in Fig. 7. Also, $\varepsilon_{x x}(1)$ is close to 1 and $\varepsilon_{x x}(2)$ has a minimum in the same region, both due to the broadened interband transition crossover with the Drude effect. That is to say, the Kerr rotation peak at $1.5 \mathrm{eV}$ is related to transitions involving spin-orbit coupling [a maximum in the numerator in Eq. (7)], along with the combination of free-carrier effects and interband transitions (a minimum in the denominator).

Notice that the $\omega \sigma_{x y}(2)$ peak is at $2.1 \mathrm{eV}$ in Ref. 15 (1.6 $\mathrm{eV}$ from our measurements). Based on this, the authors concluded that the $1.5 \mathrm{eV}$ Kerr rotation peak is dominated by the minimum in the denominator, which differs from our conclusion.

Other Kerr response peaks occur in the higher photonenergy range where the Drude effect is almost zero. The local minimum at $2.4 \mathrm{eV}$ is due to the zero crossing of $\varepsilon_{x y}(1)$ near this energy and the local minimum of $\varepsilon_{x y}(2)$ at the same energy. Both higher energy peaks in Kerr responses at $\sim 3.9$ and $\sim 4.8 \mathrm{eV}$ are related to peaks in the $\varepsilon_{x y}$ spectra (recall that $\varepsilon_{x x}$ spectra are very flat in this range). This results in the Kerr peaks in the higher energy range being determined almost entirely by the off-diagonal part of the dielectric tensor, which in turn is determined by transitions involving spin-orbit coupling.

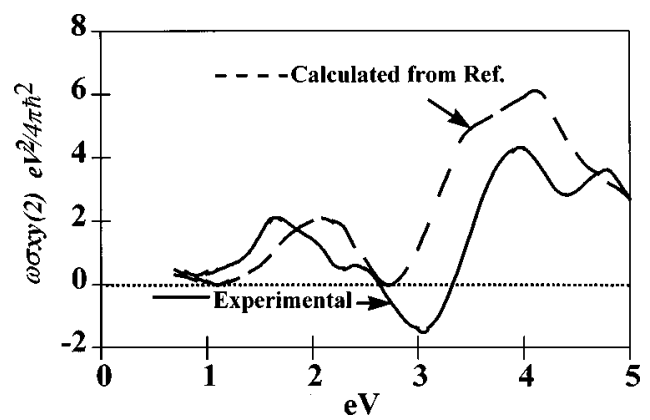

FIG. 8. Comparison of experimentally measured $\omega \sigma_{x y}(2)$ to theoretically calculated data duplicated from Ref. 15 . 


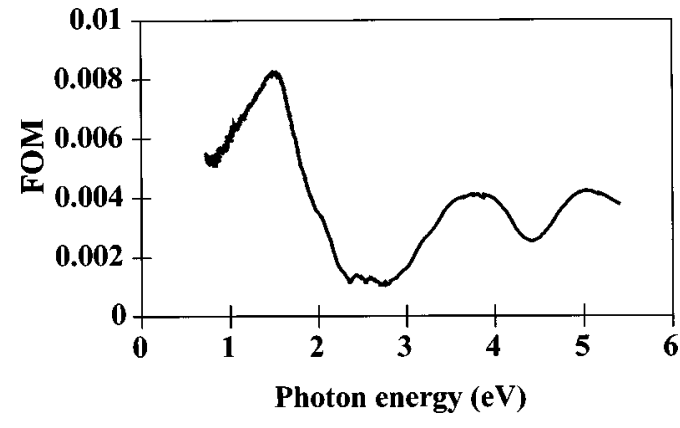

FIG. 9. Measured intrinsic figure of merit for NiMnSb.

\section{Intrinsic figure of merit for NiMnSb}

An intrinsic figure of merit (FOM) for certain materials with known dielectric tensors is given by ${ }^{30}$

$$
\mathrm{FOM}=\frac{\left|\varepsilon_{x y}\right|}{2 \cdot \operatorname{Im}\left(\varepsilon_{x x}\right)} .
$$

The intrinsic FOM spectrum was determined for $\mathrm{NiMnSb}$ using the experimental data and Eq. (9) as shown in Fig. 9. It shows that the FOM is relatively small for NiMnSb over the entire spectral range (see Ref. 30). The wavelength with the largest Kerr rotation is also that with the best FOM point at a photon energy of $\sim 1.5 \mathrm{eV}$. This peak was determined by both a maximum in the $\varepsilon_{x y}$ spectrum, as well as a minimum in the $\varepsilon_{x x}(2)$ spectrum. The other peaks in the FOM arise almost exclusively from oscillations in the $\varepsilon_{x y}$ spectra.

\section{CONCLUSIONS}

The full dielectric tensor for the ferromagnetic compound $\mathrm{NiMnSb}$ was precisely determined by the combination of $e x$ situ spectroscopic ellipsometry and magneto-optic analysis on thin film grown by MBE. This is known to be a powerful method for the study of intrinsic magneto-optic properties of multilayered structures. The diagonal dielectric component $\varepsilon_{x x}$ was well fit using the phenomenological Lorentz oscillator model with four oscillators, and a Drude term. The center energies of the oscillators are consistent with the calculated band structures for NiMnSb discussed in Ref. 15, and with the calculated minority-spin optical conductivity in Ref. 4. We find the onset energy for optical conductivity for $\mathrm{NiMnSb}$ to be smaller than reported previously (Ref. 4). The onset energy for the joint density of states is $\sim 0.6 \mathrm{eV}$ from the result of this paper. Results also show that: free-carrier effects dominate the $\varepsilon_{x x}$ spectra in the lower photon-energy range (below $1.15 \mathrm{eV}$ ); and interband transitions dominate in the higher photon-energy range (above $2.0 \mathrm{eV}$ ). More and much sharper features were found in the measured $\varepsilon_{x y}$ spectra. The highest Kerr rotation peak (also FOM peak) is due to combined contributions from: (1) crossover between the free-carrier effect and interband transitions, and (2) transitions involving spin-orbit coupling. The high Kerr and FOM peaks at higher energies result from transitions involving spin-orbit coupling.

\section{ACKNOWLEDGMENTS}

Research was supported by the National Science Foundation under Grant No. DMR-9623992, the Office of Naval Research under ONR Grant No. N00014-92-J-1847, and ONR AASERT Grant No. N00014-93-1-1204, and by the state of Nebraska through the "Research Initiative" program.
${ }^{1}$ P. G. Van Engen, K. H. J. Buschow, R. Jongebreur, and M. Erman, Appl. Phys. Lett. 42, 202 (1983).

${ }^{2}$ R. A. de Groot, F. M. Müeller, P. G. Van Engen, and K. H. J. Buschow, Phys. Rev. Lett. 50, 2024 (1983).

${ }^{3}$ R. A. de Groot, F. M. Müeller, P. G. Van Engen, and K. H. J. Buschow, J. Appl. Phys. 55, 2151 (1984).

${ }^{4}$ P. A. M. van der Heide, W. Baelde, R. A. De Groot, A. R. De Vroomen, P. G. Van Engen, and K. H. J. Buschow, J. Phys. F 15, L75 (1985).

${ }^{5}$ H. Feil and C. Haas, Phys. Rev. Lett. 58, 65 (1987).

${ }^{6}$ K. H. J. Buschow, in Ferromagnetic Materials: a Handbook on the Properties of Magnetically Ordered Substances, edited by E. P. Wohlfarth (North-Holland, Amsterdam, 1988), Vol. 4, p. 548.

${ }^{7}$ J. H. Wijngaard, C. Haas, and R. A. de Groot, Phys. Rev. B 40, 9318 (1989).

${ }^{8}$ M. J. Otto, R. A. M. Van Woerden, P. J. Van der Valk, J. Wijngaard, C. F. Van Bruggen, and C. Haas, J. Phys.: Condens. Matter 1, 2351 (1989).

${ }^{9}$ K. E. H. M. Hanssen, P. E. Mijnarends, L. P. L. M. Rabou, and K. H. J. Buschow, Phys. Rev. B 42, 1533 (1990).

${ }^{10}$ R. Kabani, M. Terada, A. Roshko, and J. S. Moodera, J. Appl. Phys. 67, 4898 (1990).

${ }^{11}$ J. S. Moodera and D. M. Mootoo, J. Appl. Phys. 76, 6101 (1994).
${ }^{12}$ X. Wang, V. P. Antropov, and B. N. Harmon, IEEE Trans. Magn. 30, 4458 (1994).

${ }^{13}$ P. M. Oppeneer and V. N. Antonov, in Spin-Orbit-InfluencedSpectroscopies of Magnetc Solids, edited by H. Ebert and G. Schütz (Springer, Berlin, 1996), p. 29.

${ }^{14}$ Yu. A. Uspenskii, E. T. Kulatov, and S. V. Khalilov, Zh. Eksp. Teor. Fiz. 107, 1708 (1995) [JETP 80, 952 (1995)].

${ }^{15}$ V. N. Antonov, P. M. Oppeneer, A. N. Yaresko, A. Ya. Perlov, and T. Kraft, Phys. Rev. B 56, 13012 (1997).

${ }^{16}$ J. Van Ek and J. M. Maclaren, Phys. Rev. B 56, R2924 (1997).

${ }^{17}$ J. A. Caballero, Y. D. Park, A. Cabbibo, J. R. Childress, F. Petroff, and R. Morel, J. Appl. Phys. 81, 2740 (1997).

${ }^{18}$ J. F. Bobo, P. R. Johnson, M. Kautzky, F. B. Mancoff, E. Tuncel, R. L. White, and B. M. Clemens, J. Appl. Phys. 81, 4164 (1997).

${ }^{19}$ C. T. Tanaka, J. Nowak, and J. S. Moodera, J. Appl. Phys. 81, 5515 (1997).

${ }^{20}$ J. A. Caballero, W. J. Geerts, J. R. Childress, F. Petroff, P. Galtier, J.-U. Thiele, and D. Weller, Appl. Phys. Lett. 71, 2382 (1997).

${ }^{21}$ P. He, W. A. McGahan, and J. A. Woollam, Proc. SPIE 1499, 401 (1991).

${ }^{22}$ W. A. McGahan, and J. A. Woollam, Appl. Phys. Commun. 9, 1 (1989). 
${ }^{23}$ X. Gao, D. W. Glenn, S. Heckens, D. W. Thompson, and J. A. Woollam. J. Appl. Phys. 82, 4525 (1997).

${ }^{24}$ Handbook of Optical Constants of Solids II, edited by E. Palik (Academic, Orlando, 1992), p. 950.

${ }^{25}$ Handbook of Optical Constants of Solids II (Ref. 24), p. 483.

${ }^{26}$ T. Lichtenstein, M.S. thesis, University of Rochester, 1979.
${ }^{27}$ F. Wooten, Optical Properties of Solids (Academic, New York, 1972).

${ }^{28}$ R. Kubo, J. Phys. Soc. Jpn. 12, 570 (1957).

${ }^{29}$ C. S. Wang and J. Callaway, Phys. Rev. B 9, 4897 (1974).

${ }^{30}$ H. Fu, Z. Yan, S. K. Lee, and M. Mansuripur, J. Appl. Phys. 78, 4076 (1995). 